MATHEMATICS OF COMPUTATION

Volume 68, Number 227, Pages 1243-1255

S 0025-5718(99)01046-7

Article electronically published on February 10, 1999

\title{
ZEROS OF 2-ADIC $L$-FUNCTIONS AND CONGRUENCES FOR CLASS NUMBERS AND FUNDAMENTAL UNITS
}

\author{
DANIEL C. SHANKS, PATRICK J. SIME, AND LAWRENCE C. WASHINGTON
}

\begin{abstract}
We study the imaginary quadratic fields such that the Iwasawa $\lambda_{2}$-invariant equals 1 , obtaining information on zeros of 2 -adic $L$-functions and relating this to congruences for fundamental units and class numbers.
\end{abstract}

This paper explores the interplay between zeros of 2-adic $L$-functions and congruences for fundamental units and class numbers of quadratic fields. An underlying motivation was to study the distribution of zeros of 2 -adic $L$-functions, the basic philosophy being that the location of the zeros causes restrictions on the 2 -adic behavior of the class numbers and fundamental units of real quadratic fields. Though the predicted restrictions involved the unit and class number together, numerical computations (we used PARI) revealed definite patterns for the unit and class number separately, which we were then able to prove. Several of these congruences are classical, but some of them seem to be new.

We use the information obtained to study the distribution of the zeros, in particular their distances from 1 and 0 . In a previous paper [14], one of us showed that, if $\left(2^{p}+1\right) / 3$ is prime infinitely often, then it is possible to have zeros of 2 -adic $L$ functions arbitrarily close to $s=1$. Recently, Morain [7] showed that $\left(2^{12391}+1\right) / 3$ is prime, which yields a 2 -adic $L$-function with a zero $\beta$ satisfying $|\beta-1|_{2}=2^{-6194}$ (see the discussion following Theorem 5).

In previous papers [12], [15], one of the present authors studied zeros of 3-adic $L$-functions in a somewhat similar approach. However, the advantage of using 2adic $L$-functions for quadratic fields $\mathbb{Q}(\sqrt{m})$ is that not only is the number of zeros bounded by $\lambda^{-}$, the Iwasawa invariant for the cyclotomic $\mathbb{Z}_{2}$-extension of $\mathbb{Q}(\sqrt{-m})$, but also there is a simple formula for $\lambda^{-}$due to Y. Kida [6] and B. Ferrero [4]. This allows us to keep the number of zeros under control. In fact, throughout the present paper we restrict ourselves to the case $\lambda^{-}=1$, so we are dealing with at most one zero.

\section{2-ADIC $L$-FUNCTIONS}

Let $\chi$ be the non-trivial Dirichlet character associated to the real quadratic field $\mathbb{Q}(\sqrt{m})$, where $m$ is taken to be squarefree. The 2 -adic $L$-function $L_{2}(s, \chi)$ satisfies

Received by the editor October 14, 1997.

1991 Mathematics Subject Classification. Primary 11R11; Secondary 11S40.

Key words and phrases. Quadratic fields, $p$-adic $L$-functions.

The third author was partially supported by a grant from NSA, and also thanks the Institute for Advanced Study for its hospitality during part of the preparation of this paper.

(C)1999 American Mathematical Society 


$$
L_{2}(1-n, \chi)=-\left(1-\chi \omega^{-n}(2) 2^{n-1}\right) \frac{B_{n, \chi \omega^{-n}}}{n}
$$

for all $n \geq 1$, where $\omega$ is the non-trivial character $\bmod 4$ and $B_{n, \chi \omega^{-n}}$ is a generalized Bernoulli number (for more on $p$-adic $L$-functions, see [13]). The 2 -adic class number formula states that

$$
L_{2}(1, \chi)=\left(1-\frac{\chi(2)}{2}\right) \frac{2 h^{+} \log _{2} \epsilon}{\sqrt{d}},
$$

where $h^{+}, \epsilon$, and $d$ are the class number, fundamental unit, and discriminant of $\mathbb{Q}(\sqrt{m})$, and $\log _{2}$ is the 2-adic logarithm. Iwasawa has shown that there is a power series $g(T)=g(T, \chi) \in \mathbb{Z}_{2}[[T]]$ such that

$$
L_{2}(s, \chi)=2 g\left((1+4)^{s}-1\right) \text {. }
$$

The Weierstrass preparation theorem says that there is a factorization

$$
g(T)=P(T) U(T)
$$

where $P(T)$ is a distinguished polynomial and $U(T)$ is invertible in $\mathbb{Z}_{2}[[T]]$. The degree of $P(T)$ is $\lambda^{-}$, the Iwasawa invariant for the cyclotomic $\mathbb{Z}_{2}$-extension of $\mathbb{Q}(\sqrt{-m})$. Note that $\mathbb{Q}(\sqrt{-m})$ corresponds to the character $\chi \omega^{-1}$.

Proposition (Kida [6], Ferrero [4]). Let $m>4$ be squarefree and let $\lambda^{-}$be the Iwasawa invariant for the cyclotomic $\mathbb{Z}_{2}$-extension of $\mathbb{Q}(\sqrt{-m})$. Then

$$
\lambda^{-}=-1+\sum_{\substack{p \mid m \\ p \text { odd }}} \frac{1}{8}\left[p^{2}-1\right]_{2},
$$

where $[n]_{2}=2^{v_{2}(n)}$ is the largest power of 2 dividing $n$.

Corollary. (a) $\lambda^{-}=0$ if and only if $m=p$ or $2 p$, where $p \equiv \pm 3(\bmod 8)$ is prime.

(b) $\lambda^{-}=1$ if and only if $m$ or $m / 2$ is one of the following:

1. $p$, with $p \equiv \pm 7(\bmod 16)$

2. $p q$, with $p, q \equiv \pm 3(\bmod 8)$.

Let $g(T)=b_{0}+b_{1} T+b_{2} T^{2}+\cdots$. Recall that $\lambda^{-}$is the index of the first coefficient $b_{i}$ that is not divisible by 2 . Therefore $\lambda^{-}=0$ if and only if $2 \nmid b_{0}$, and $\lambda^{-}=1$ if and only if $2 \mid b_{0}$ and $2 \nmid b_{1}$. Note that

$$
2 b_{0}=2 g(0)=L_{2}(0, \chi)=-\left(1-\chi \omega^{-1}(2)\right) B_{1, \chi \omega^{-1}},
$$

so $b_{0}=0$ if and only if $\chi \omega^{-1}(2)=1$, which happens if and only if $m \equiv 7(\bmod 8)$. In this case we say that $L_{2}(s, \chi)$ has a trivial zero.

Let $h^{-}$be the class number of $\mathbb{Q}(\sqrt{-m})$. It is well-known that $h^{-}=-B_{1, \chi \omega^{-1}}$, so

$$
b_{0}=\frac{1}{2}\left(1-\chi \omega^{-1}(2)\right) h^{-} .
$$

We can also consider a power series $f(T)=g\left(\frac{5}{1+T}-1\right)=a_{0}+a_{1} T+\cdots$. Then

$$
L_{2}(s, \chi)=2 f\left((1+4)^{1-s}-1\right)
$$

and $a_{0}=\frac{1}{2} L_{2}(1, \chi)$. Since $T \mapsto \frac{5}{1+T}-1$ is an invertible change of variables over $\mathbb{Z}_{2}$, we find that $\lambda^{-}$is the index $i$ of the first odd coefficient $a_{i}$. 
Theorem 1. Assume $\lambda^{-}=1$.

1. $v_{2}\left(b_{0}\right) \geq 1$ and $v_{2}\left(L_{2}(1, \chi)\right) \geq 2$.

2. $L_{2}(s, \chi)$ has a zero $\beta \in \mathbb{Z}_{2}$ if and only if $v_{2}\left(b_{0}\right) \geq 2$, and if and only if $v_{2}\left(L_{2}(1, \chi)\right) \geq 3$.

3. If $\beta$ exists, then $v_{2}(\beta-1) \geq 1$ if and only if $v_{2}\left(b_{0}\right)=2$, and $v_{2}(\beta) \geq 1$ if and only if $v_{2}\left(L_{2}(1, \chi)\right)=3$.

4. If $\beta$ exists, then $v_{2}\left(L_{2}(1, \chi)\right)=v_{2}(\beta-1)+3$ and $v_{2}\left(b_{0}\right)=v_{2}(\beta)+2$.

Remark. Let $\psi$ be the quadratic character corresponding to $\mathbb{Q}(\sqrt{2})$. It follows from the work of Childress and Gold [2] that if $\lambda^{-}=1$ and $L_{2}(s, \chi)$ has no zero in $\mathbb{Z}_{2}$, then the zero appears one step up (if $2 \nmid m$ ) the $\mathbb{Z}_{2}$-extension of the quadratic field $\mathbb{Q}(\sqrt{m})$; namely, $L_{2}(s, \psi \chi)=2 g\left(-(1+4)^{s}-1\right)$ has a zero. This can be seen in the proof below, since in this case $(1+4)^{s}+1=-\alpha$ has a solution $s=\beta$.

Proof. The assumption that $\lambda^{-}=1$ yields (1). We have $\operatorname{deg} P(T)=\lambda^{-}=1$, so $g(T)=(T-\alpha) U(T)$ with $\alpha \in 2 \mathbb{Z}_{2}$. Since $U(0) \in \mathbb{Z}_{2}^{\times}$, it follows that $v_{2}(\alpha)=v_{2}\left(b_{0}\right)$. If $L_{2}(\beta, \chi)=0$, then $(1+4)^{\beta}-1=\alpha$, so $\alpha \equiv 0(\bmod 4)$, hence $v_{2}\left(b_{0}\right) \geq 2$. Moreover, $v_{2}(\alpha)=2$ if and only if $\beta$ is odd. Suppose now that $v_{2}\left(b_{0}\right) \geq 2$. Then $\beta=\log _{2}(1+\alpha) / \log _{2}(1+4)$ is a root of $L_{2}(s, \chi)$. The same argument applied to $f(T)$ completes the proofs of (2) and (3).

To prove (4), let $\beta$ and $\alpha$ be as above. Then

$$
\begin{aligned}
L_{2}(1, \chi) & =L_{2}(1, \chi)-L_{2}(\beta, \chi) \\
& =2 g(4)-2 g(\alpha) \\
& =2\left(b_{1}(4-\alpha)+b_{2}\left(4^{2}-\alpha^{2}\right)+\cdots\right) \\
& =2(4-\alpha)\left(b_{1}+b_{2}(4+\alpha)+\cdots\right) \\
& \equiv 2(4-\alpha) \quad(\bmod 4(4-\alpha)),
\end{aligned}
$$

since $b_{1}$ is odd and $\alpha \equiv 0(\bmod 4)$. But $\alpha-4=(1+4)\left((1+4)^{\beta-1}-1\right) \equiv 4(\beta-1)$ $(\bmod 8(\beta-1))$, so the first part of $(4)$ follows. The second part follows from the same argument applied to $f(T)$.

The above theorem expresses quantitatively the principle that a zero close to 1 causes $L_{2}(1, \chi)$ to be small. This can happen only if either $h^{+}$or $\log _{2} \epsilon$ is divisible by a high power of 2 . On the other hand, if $h^{-}$is divisible by a high power of 2 then $\beta \in 2 \mathbb{Z}_{2}$, which of course says that if $L_{2}(0, \chi)$ is small then $\beta$ is forced to be near 0 . Theorem 1 has the following interesting consequence.

Corollary. Assume $\lambda^{-}=1$. If $v_{2}\left(b_{0}\right) \geq 3$ then $v_{2}\left(L_{2}(1, \chi)\right)=3$. If $v_{2}\left(L_{2}(1, \chi)\right) \geq$ 4 then $v_{2}\left(b_{0}\right)=2$.

In particular, this implies, in the case $\lambda^{-}=1$, that when $h^{-}$is divisible by a high power of 2 , or if $\chi \omega^{-1}(2)=1$, then $v_{2}\left(h^{+}\right)$and $v_{2}\left(\log _{2} \epsilon\right)$ are bounded. Also, if $v_{2}\left(h^{+}\right)$or $v_{2}\left(\log _{2} \epsilon\right)$ is large, then $v_{2}\left(h^{-}\right)$is bounded (as long as the Euler factor does not cause a trivial zero. In the next section, we investigate this phenomenon. Our point of view is to start with $v_{2}\left(h^{-}\right)$and see what restrictions are imposed on $v_{2}\left(h^{+}\right)$and $v_{2}\left(\log _{2} \epsilon\right)$. Of course, we could similarly start with $v_{2}\left(h^{+}\right)+v_{2}\left(\log _{2} \epsilon\right)$ and study the restrictions imposed on $v_{2}\left(h^{-}\right)$. In fact, in Theorem 4(2), Theorem $5(3)$, Theorem $6(2)$, and Theorem $7(\mathrm{~b})(1)$, we have $v_{2}\left(h^{-}\right)=3$, but only inequalities for what happens with $v_{2}\left(h^{+}\right)$and $v_{2}\left(\log _{2} \epsilon\right)$. This is because the cause and effect are reversed: $h^{+}$and $\epsilon$ are causing the restriction $v_{2}\left(h^{-}\right)=3$. 


\section{Congruences}

The above corollary implies that if $v_{2}\left(b_{0}\right) \geq 3$ then we have 2 -adic restrictions on $h^{+}$and $\epsilon$. In this section we investigate this phenomenon.

Throughout, $p$ and $q$ will always denote primes. We let

$h^{+}=$class number of $\mathbb{Q}(\sqrt{m})$,

$h_{0}^{+}=$narrow class number of $\mathbb{Q}(\sqrt{m})$,

$h^{-}=$the class number of $\mathbb{Q}(\sqrt{-m})$,

$\epsilon=a+b \sqrt{m}=$ the fundamental unit of $\mathbb{Q}(\sqrt{m})$,

$\epsilon^{2}=A+B \sqrt{m}$.

Recall that $h^{+}=h_{0}^{+}$if $\epsilon$ has norm -1 , and $h^{+}=h_{0}^{+} / 2$ if $\epsilon$ has norm +1 .

We will often need the fact that when $\epsilon$ has norm +1 ,

$$
\sqrt{\epsilon}=\sqrt{\frac{1}{2}(a+1)}+\sqrt{\frac{1}{2}(a-1)} .
$$

In particular, $\frac{1}{2}(a \pm 1)$ cannot both be of the form $m r^{2}$ or $r^{2}$ with $r \in \mathbb{Q}$, since otherwise $\sqrt{\epsilon}$ would be in $\mathbb{Q}(\sqrt{m})$.

Another fact we will use often is that if $x \equiv \pm 1\left(\bmod 2^{3 / 2}\right)$ then $v_{2}\left(\log _{2} x\right)=$ $v_{2}(x \mp 1)$.

We start with the case of a trivial zero, so $b_{0}=0$. Since we must have $m \equiv 7$ $(\bmod 8)$, we have either $m=p \equiv 7(\bmod 16)$ or $m=p q$ with $p \equiv 3(\bmod 8)$ and $q \equiv 5(\bmod 8)$. The following result does not seem to be well-known; we did not find it in the literature.

Theorem 2. (a) If $m=p \equiv 7(\bmod 16)$, then

$$
\begin{gathered}
v_{2}\left(h^{+}\right)=0, \quad v_{2}\left(\log _{2} \epsilon\right)=3, \quad v_{2}\left(h^{-}\right)=0, \\
a \equiv 8 \quad(\bmod 16), \quad b \equiv \pm 3 \quad(\bmod 8) .
\end{gathered}
$$

(b) If $m=p q$ with $p \equiv 3(\bmod 8)$ and $q \equiv 5(\bmod 8)$, then

$$
v_{2}\left(h^{+}\right)=1, \quad v_{2}\left(\log _{2} \epsilon\right)=2, \quad A \equiv \pm 31 \quad(\bmod 64), \quad B \equiv 8 \quad(\bmod 16) .
$$

Remark. In part (b), $v_{2}\left(h^{-}\right)$is not constant. For example, when $m=15, h^{-}=2$ and when $m=39, h^{-}=4$.

Proof. (a) The fact that $h^{-}$is odd is classical. We have $(a+1)(a-1)=p b^{2}$. If $\operatorname{gcd}(a+1, a-1)=2$ then one of $(a \pm 1) / 2$ is a square and the other is $p$ times a square. Therefore $\sqrt{\epsilon}=\sqrt{\frac{1}{2}(a+1)}+\sqrt{\frac{1}{2}(a-1)} \in \mathbb{Q}(\sqrt{p})$, which is impossible. Therefore gcd $=1$. If $a-1=r^{2}$ and $a+1=s^{2} p$ for integers $r, s$, then $s^{2} p-r^{2}=2$, which is impossible mod 8. Therefore $a+1=r^{2}$ and $a-1=s^{2} p$, so $r^{2}-s^{2} p=2$. Clearly $r, s$ are odd. Since $r^{2} \equiv 2$ modulo each prime factor of $s$, each such factor must be $\pm 1 \bmod 8$, so $s \equiv \pm 1(\bmod 8)$. Therefore $a=1+s^{2} p \equiv 8(\bmod 16)$. Since $a^{2}-p b^{2}=1$, we must have $b \equiv \pm 3(\bmod 8)$. Therefore $\epsilon^{2}=2 a^{2}-1+2 a b \sqrt{p} \equiv$ $-1+16 \sqrt{p}(\bmod 32)$, so $v_{2}\left(\log _{2} \epsilon^{2}\right)=4$ and $v_{2}\left(\log _{2} \epsilon\right)=3$. Since $3=v_{2}\left(L_{2}(1, \chi)\right)=$ $v_{2}\left(h^{+}\right)+v_{2}\left(\log _{2} \epsilon\right)$, we have $v_{2}\left(h^{+}\right)=0$. 
Part (a) can also be proved as follows using quadratic forms (in fact, this was our original proof). The principal cycle for the quadratic form $x^{2}-p y^{2}$ has even length, since the fundamental unit $\epsilon$ has positive norm. Halfway through the cycle is an ambiguous form $\alpha x^{2}+\beta x y+\gamma y^{2}$ with $\alpha= \pm 2$. This means that \pm 2 is represented by the original form: $\pm 2=x^{2}-p y^{2}$. Congruences exclude -2 . Moreover, $(x+y \sqrt{p})^{2}=$ $2 \epsilon$, which yields $a=\left(x^{2}+p y^{2}\right) / 2=x^{2}-1$ and $a-1=x^{2}-2=p y^{2}$. The proof now proceeds as above.

Since many modern readers might be somewhat unfamiliar with quadratic forms, we now restate and justify what we just did in terms of the equivalent statements for continued fractions (and we apologize to the first author). First we need the following lemma. Surely it is well known, but since we did not find a reference we prove it.

Lemma. Let $d>1$ be squarefree, let $\sqrt{d}=\left[a_{0} ; \overline{a_{1}, \ldots, a_{n}, 2 a_{0}}\right]$ be the continued fraction expansion of $\sqrt{d}$, let $p_{m} / q_{m}=\left[a_{0}, \ldots, a_{m}\right]$ be the mth convergent, and let $p_{-1}=1$ and $q_{-1}=0$. Let $\epsilon=p_{n}+q_{n} \sqrt{d}$ be the fundamental unit of $\mathbb{Q}(\sqrt{d})$. Then

$$
\frac{p_{n-r}+q_{n-r} \sqrt{d}}{p_{r-1}-q_{r-1} \sqrt{d}}=(-1)^{r+1} \epsilon
$$

for $0 \leq r \leq n+1$.

Proof. The case $r=0$ is the definition of $\epsilon$. It suffices to prove that

$$
\frac{p_{n-r}+q_{n-r} \sqrt{d}}{p_{r-1}-q_{r-1} \sqrt{d}}=-\frac{p_{n-r-1}+q_{n-r-1} \sqrt{d}}{p_{r}-q_{r} \sqrt{d}}
$$

for $0 \leq r \leq n$, which is equivalent to the pair of equations

$$
\begin{gathered}
p_{n-r} p_{r}-q_{n-r} q_{r} d+p_{n-r-1} p_{r-1}-q_{n-r-1} q_{r-1} d=0, \\
q_{n-r} p_{r}-p_{n-r} q_{r}+q_{n-r-1} p_{r-1}-p_{n-r-1} q_{r-1}=0 .
\end{gathered}
$$

The case $r=0$ is equivalent to

$$
\sqrt{d}=\left(\left(\sqrt{d}+a_{0}\right) p_{n}+p_{n-1}\right) /\left(\left(\sqrt{d}+a_{0}\right) q_{n}+q_{n-1}\right),
$$

which is well known [9, p. 114]. Assuming that the case $r$ for the first equation has been proved, we use $p_{n-r}=a_{n-r} p_{n-r-1}+p_{n-r-2}$, and similarly for $q_{n-r}, p_{r+1}, q_{r+1}$, to rewrite the first equation as

$$
\begin{aligned}
\left(a_{n-r} p_{n-r-1}\right. & \left.+p_{n-r-2}\right) p_{r}-\left(a_{n-r} q_{n-r-1}+q_{n-r-2}\right) q_{r} d \\
& +p_{n-r-1}\left(p_{r+1}-a_{r+1} p_{r}\right)-q_{n-r-1}\left(q_{r+1}-a_{r+1} q_{r}\right) d=0 .
\end{aligned}
$$

Using the fact that $a_{r+1}=a_{n-r}$ and canceling the appropriate terms yields the first equation with $r+1$ in place of $r$. The second equation is treated similarly.

We now return to the case $p \equiv 7(\bmod 16)$ Since the fundamental unit $\epsilon=$ $a+b \sqrt{p}$ has positive norm, the value of $n$ in the lemma must be odd. Letting $r=(n+1) / 2$ and $x=p_{r-1}, y=q_{r-1}$, we have

$$
\frac{x+y \sqrt{p}}{x-y \sqrt{p}}= \pm \epsilon
$$

Therefore the primitive ideal $(x+y \sqrt{p})$ must be a product of ramified primes. Since only the primes above 2 and $p$ ramify, we have $\pm\left(x^{2}-p y^{2}\right)=1,2, p, 2 p$. But 1 is not possible since $r-1<n$. Since $\left|x^{2}-p y^{2}\right|<p$, we must have $x^{2}-p y^{2}= \pm 2=2$ (the last equality obtained because of congruences mod 8 ). Moreover, multiplying the 
numerator and denominator of the above equation by $x+y \sqrt{p}$ yields $(x+y \sqrt{p})^{2}=$ $2 \epsilon$. This justifies the steps used in the second proof above.

(b) By Theorem 1, we have $3=v_{2}\left(L_{2}(1, \chi)\right)=v_{2}\left(h^{+}\right)+v_{2}\left(\log _{2} \epsilon\right)$. Since three primes divide the discriminant of $\mathbb{Q}(\sqrt{m}), 4 \mid h_{0}^{+}$, so $2 \mid h^{+}$. Therefore $v_{2}\left(\log _{2} \epsilon\right) \leq 2$.

If $a$ is even, then it is easy to see that $4 \mid a$ and hence $-p q b^{2} \equiv 1(\bmod 16)$. Therefore $\epsilon^{2}=a^{2}+p q b^{2}+2 a b \sqrt{p q} \equiv-1+2 a b \sqrt{p q}(\bmod 16)$, so $\log _{2}\left(\epsilon^{2}\right) \equiv 0$ $(\bmod 8)$. Therefore $v_{2}\left(\log _{2} \epsilon\right) \geq 2$, and we have equality. It follows that $v_{2}(a)=2$ and also $v_{2}\left(h^{+}\right)=1$. Moreover, $v_{2}\left(\log _{2} \epsilon\right)=2$ implies that $B=2 a b \equiv 8(\bmod 16)$. Note that $A \equiv-1(\bmod 16)$.

If $a$ is odd, then we must have $4 \mid b$, hence $a^{2} \equiv 1(\bmod 16)$. Therefore $\epsilon^{2} \equiv$ $1+2 a b \sqrt{p q}(\bmod 16)$, so $v_{2}\left(\log _{2} \epsilon\right) \geq 2$. Therefore we have equality and $v_{2}(b)=2$, $v_{2}\left(h^{+}\right)=1$. Also, $A \equiv 1(\bmod 16)$ and $B \equiv 8(\bmod 16)$.

Since $v_{2}\left(A^{2}-1\right)=v_{2}\left(B^{2}\right)=6$ and we already have $A \equiv \pm 1(\bmod 16)$, we have $A \equiv \pm 31(\bmod 64)$.

We now systematically examine the cases where $\lambda^{-}=1$ and the zero, if it exists, is non-trivial. We first treat the case where $m$ is even, since then $\chi(2)=$ $\chi \omega^{-1}(2)=0$, so the Euler factors disappear in the expressions for $L_{2}(1, \chi)$ and $L_{2}(0, \chi)$. Therefore

in this case.

$$
v_{2}\left(L_{2}(1, \chi)\right)=v_{2}\left(h^{+}\right)+v_{2}\left(\log _{2} \epsilon\right)-\frac{1}{2}
$$

Theorem 3. If $m=2 p$ with $p \equiv 7(\bmod 16)$, then

$$
\begin{gathered}
v_{2}\left(h^{+}\right)=0, \quad v_{2}\left(\log _{2} \epsilon\right)=\frac{5}{2}, \quad v_{2}\left(h^{-}\right)=2, \quad L_{2}(s, \chi) \text { has no zero in } \mathbb{Z}_{2} \\
a \equiv 15 \quad(\bmod 128), \quad b \equiv 4 \quad(\bmod 8) .
\end{gathered}
$$

Proof. $v_{2}\left(h^{-}\right)=2$ by [5, Thm. 4 and p. 596]. Therefore $v_{2}\left(b_{0}\right)=1$. By Theorem $1, L_{2}(s, \chi)$ has no zero in $\mathbb{Z}_{2}$ and $v_{2}\left(L_{2}(1, \chi)\right)=2$.

We have $(a+1)(a-1)=2 p b^{2}$. Since $a$ must be odd and $b$ must be even, $(a+1) / 2$ is a square times $1,2, p$, or $2 p$. The first and last on this list would imply that $\sqrt{\epsilon} \in \mathbb{Q}(\sqrt{2 p})$, so 2 and $p$ remain. If $(a+1) / 2=p r^{2}$, then $(a-1) / 2=2 s^{2}$, so $p r^{2}-2 s^{2}=1$, which is impossible $\bmod 8$. Therefore $(a+1) / 2=2 r^{2}$ and $(a-1) / 2=p s^{2}$, so $2 r^{2}-p s^{2}=1$. Since 2 is a square modulo each prime factor of $s$, we have $s \equiv \pm 1(\bmod 8)$, hence $s^{2} \equiv 1(\bmod 16)$. It follows that $r \equiv 2$ $(\bmod 4)$ and $a=4 r^{2}-1 \equiv 15(\bmod 128)$. This implies that $b \equiv 4(\bmod 8)$ and $v_{2}\left(\log _{2} \epsilon\right)=\frac{5}{2}$. Since $2=v_{2}\left(L_{2}(1, \chi)\right)=v_{2}\left(h^{+}\right)+v_{2}\left(\log _{2} \epsilon\right)-\frac{1}{2}$, we have $v_{2}\left(h^{+}\right)=0$.

Theorem 4. Suppose $m=2 p$ with $p \equiv 9(\bmod 16)$. Then $v_{2}\left(h^{-}\right) \geq 2$.

1. If $v_{2}\left(h^{-}\right)=2$ then $\operatorname{Norm} \epsilon=-1$ and

$$
\begin{gathered}
v_{2}\left(h^{+}\right)=2, \quad v_{2}\left(\log _{2} \epsilon\right)=\frac{1}{2}, \quad L_{2}(s, \chi) \text { has no zero in } \mathbb{Z}_{2}, \\
a \equiv \pm 1 \quad(\bmod 8), \quad b \equiv 1 \quad(\bmod 4) .
\end{gathered}
$$

2. If $v_{2}\left(h^{-}\right)=3$ then

$$
v_{2}\left(h^{+}\right)=1, \quad \frac{1}{2} v_{2}(a-1)=v_{2}(b)=v_{2}\left(\log _{2} \epsilon\right)-\frac{1}{2}=v_{2}(\beta-1)+2 \geq 3 .
$$


3. If $v_{2}\left(h^{-}\right) \geq 4$, then

$$
\begin{gathered}
v_{2}\left(h^{+}\right)=1, \quad v_{2}\left(\log _{2} \epsilon\right)=\frac{5}{2}, \quad v_{2}(\beta)=v_{2}\left(h^{-}\right)-3 \geq 1, \\
a \equiv 17 \quad(\bmod 128), \quad b \equiv 4 \quad(\bmod 8) .
\end{gathered}
$$

Proof. By [5, Thm. 1 and p. 596], $v_{2}\left(h_{0}^{+}\right)=2$. The valuation of $h^{+}$therefore depends on the sign of the norm of $\epsilon$.

Suppose $\operatorname{Norm}(\epsilon)=+1$. Note that $a^{2}-2 p b^{2}=1$ implies that $a$ is odd and $b$ is even. If $(a+1) / 2=2 r^{2}$ and $(a-1) / 2=p s^{2}$, then $2 r^{2}-p s^{2}=1$, so 2 is a square mod all prime divisors of $s$. As in Theorem 3 , we find that $s^{2} \equiv 1(\bmod 16)$ and $r^{2} \equiv 5(\bmod 8)$, which is impossible. Therefore $(a+1) / 2=p r^{2}$ and $(a-1) / 2=2 s^{2}$, and $p r^{2}-2 s^{2}=1$. It follows that $s$ is even, hence $a=1+4 s^{2} \equiv 1(\bmod 16)$. This implies that $b \equiv 0(\bmod 4)$, so $v_{2}\left(\log _{2} \epsilon\right) \geq \frac{5}{2}$.

As in Theorem 3 , if $v_{2}\left(h^{-}\right)=2$ then $L_{2}(s, \chi)$ has no zero in $\mathbb{Z}_{2}$ and $v_{2}\left(L_{2}(1, \chi)\right)=$ 2 , so $2=v_{2}\left(h^{+}\right)+v_{2}\left(\log _{2} \epsilon\right)-\frac{1}{2}$. Since $4 \mid h_{0}^{+}$, we have $v_{2}\left(h^{+}\right) \geq 1$, hence $v_{2}\left(\log _{2} \epsilon\right) \leq \frac{3}{2}$. From the above we see that we must have $\operatorname{Norm}(\epsilon)=-1$ (this could also be obtained from [8, Thm. 2 (i) and Thm. 5] or [5, Thm. 3 and Prop. $2]$ ). Since $a$ and $b$ must be odd and $\epsilon^{2}=A+B \sqrt{2 p}=2 a^{2}+1+2 a b \sqrt{2 p}$, we have $A \equiv 3(\bmod 16)$ and $B \equiv 2(\bmod 4)$. Therefore $\epsilon^{2} \equiv-1+2 \sqrt{2 p}(\bmod 4)$, so $v_{2}\left(\log _{2}\left(\epsilon^{2}\right)\right)=\frac{3}{2}$ and $v_{2}(\log \epsilon)=\frac{1}{2}$. It follows that in this case we are forced to have $v_{2}\left(h^{+}\right)=2$ (this also follows from $v_{2}\left(h_{0}^{+}\right)=2$ ). Since $a^{2}-2 p b^{2}=-1$, we see that -1 is a square modulo $b$, so $b \equiv 1(\bmod 4)$. Moreover, $a^{2}=2 p b^{2}-1 \equiv 1$ $(\bmod 16)$ yields $a \equiv \pm 1(\bmod 8)$.

If $v_{2}\left(h^{-}\right) \geq 3$ then $t^{2}-2 p u^{2}=-2$ has an integral solution by [5, Thm. 3 and Prop. $2(\beta)]$. By [5, p. 600], $\operatorname{Norm}(\epsilon)=+1$, so $v_{2}\left(h^{+}\right)=v_{2}\left(h_{0}^{+} / 2\right)=1$.

If $v_{2}\left(h^{-}\right) \geq 4$, then Theorem 1 implies that $v_{2}(\beta-1)=0$ and $v_{2}\left(L_{2}(1, \chi)\right)=3$. Therefore $v_{2}\left(\log _{2} \epsilon\right)=\frac{5}{2}$. The above implies that $b \equiv 4(\bmod 8)$. Since $a^{2}=$ $2 p b^{2}+1 \equiv 33(\bmod 256)$, and since $a \equiv 1(\bmod 16)$, we have $a \equiv 17(\bmod 128)$.

If $v_{2}\left(h^{-}\right)=3$, then $4 \leq v_{2}(\beta-1)+3=v_{2}\left(L_{2}(1, \chi)\right)=\frac{1}{2}+v_{2}\left(\log _{2} \epsilon\right)$. Since $v_{2}(a-1)=2 v_{2}(b)$, we have $v_{2}\left(\log _{2} \epsilon\right)=v_{2}(b)+\frac{1}{2}$.

Theorem 5. Suppose $m=2 p q$ with $p \equiv \pm 3(\bmod 8)$ and $q \equiv \pm 3(\bmod 8)$.

1. If $p \equiv q \equiv 5(\bmod 8)$ then

$$
\begin{gathered}
v_{2}\left(h^{+}\right)=2, \quad v_{2}\left(\log _{2} \epsilon\right)=\frac{1}{2}, \quad \operatorname{Norm} \epsilon=-1, \quad v_{2}\left(h^{-}\right)=2, \\
L_{2}(s, \chi) \text { has no zero in } \mathbb{Z}_{2}, \quad a \equiv \pm 1 \quad(\bmod 8), \quad b \equiv 1 \quad(\bmod 4) .
\end{gathered}
$$

2. If $p \equiv 3(\bmod 8)$ and $q \equiv 5(\bmod 8)$, then

$$
\begin{gathered}
v_{2}\left(h^{+}\right)=1, \quad v_{2}\left(\log _{2} \epsilon\right)=\frac{3}{2}, \quad v_{2}\left(h^{-}\right)=2, \quad L_{2}(s, \chi) \text { has no zero in } \mathbb{Z}_{2}, \\
a \equiv \pm 11 \quad(\bmod 32), \quad b \equiv 2 \quad(\bmod 4) .
\end{gathered}
$$

3. If $p \equiv q \equiv 3(\bmod 8)$ then $v_{2}\left(h^{-}\right) \geq 3$ and $v_{2}\left(h^{+}\right)=1$. If $v_{2}\left(h^{-}\right)=3$ then

$$
3 \leq \frac{1}{2} v_{2}(a-1)=v_{2}(b)=v_{2}\left(\log _{2} \epsilon\right)-\frac{1}{2}=v_{2}(\beta-1)+2 .
$$




$$
\begin{aligned}
& \text { If } v_{2}\left(h^{-}\right) \geq 4 \text {, then } \\
& \qquad \begin{aligned}
2=\frac{1}{2} v_{2}(a-1) & =v_{2}(b)=v_{2}\left(\log _{2} \epsilon\right)-\frac{1}{2}, \\
v_{2}(\beta) & =v_{2}\left(h^{-}\right)-3 \geq 1 .
\end{aligned}
\end{aligned}
$$

Proof. Consider first the case where $p \equiv q \equiv 5(\bmod 8)$. Suppose $a^{2}-2 p q b^{2}=+1$. Then $a$ is odd and $b$ is even. If $(a+1) / 2=2 p r^{2}$ and $(a-1) / 2=q s^{2}$, then $2 p r^{2}-q s^{2}=1$, which is impossible mod 8. If $(a+1) / 2=p r^{2}$ and $(a-1) / 2=2 q s^{2}$, then $p r^{2}-2 q s^{2}=1$, which is again impossible mod 8. If $(a \pm 1) / 2=2 r^{2}$ and $(a \mp 1) / 2=p q s^{2}$, then $2 r^{2}-p q s^{2}= \pm 1$, which implies that \pm 2 is a quadratic residue mod $p$, which is not the case (of course, we could switch the roles of $p$ and $q$ in the above, with similar results). The remaining possibilities imply that $\sqrt{\epsilon} \in \mathbb{Q}(\sqrt{2 p q})$, so $\epsilon$ must have negative norm. Therefore $a^{2}-2 p q b^{2}=-1$, which implies that $b$ is odd. Consequently, $a^{2} \equiv 1(\bmod 16)$, so $a \equiv \pm 1(\bmod 8)$. Since -1 is a square modulo each prime factor of $b$, we have $b \equiv 1(\bmod 4)$. Since $\epsilon^{2}=A+B \sqrt{2 p q}$ with $A \equiv 3(\bmod 16)$ and $B \equiv 2(\bmod 4)$, we obtain $v_{2}\left(\log _{2} \epsilon\right)=v_{2}\left(\log _{2}\left(\epsilon^{2}\right)\right)-1=\frac{1}{2}$. By $\left[10\right.$, p. 191], we have $v_{2}\left(h^{-}\right)=2$. Therefore $L_{2}(s, \chi)$ has no zero $\beta \in \mathbb{Z}_{2}$ and $2=v_{2}\left(L_{2}(1, \chi)\right)=v_{2}\left(h^{+}\right)+v_{2}\left(\log _{2} \epsilon\right)-\frac{1}{2}$. Therefore $v_{2}\left(h^{+}\right)=2$.

If $p \equiv 3(\bmod 8)$ and $q \equiv 5(\bmod 8)$, then $v_{2}\left(h^{-}\right)=2$ by [10, p. 191]. Therefore $L_{2}(s, \chi)$ has no zero $\beta \in \mathbb{Z}_{2}$ and $2=v_{2}\left(L_{2}(1, \chi)\right)=v_{2}\left(h^{+}\right)+v_{2}\left(\log _{2} \epsilon\right)-\frac{1}{2}$. Since $a^{2}-2 p q b^{2}=1$, we have $a$ odd and $b$ even. The possibilities $(a+1) / 2=q r^{2}$ and $=2 q r^{2}$ are easily eliminated by congruences $\bmod 8$. If $(a \pm 1) / 2=2 r^{2}$ and $(a \mp 1) / 2=p q s^{2}$, then $2 r^{2}-p q s^{2}= \pm 1$ implies that \pm 2 is a quadratic residue $\bmod q$, which is not the case. If $(a+1) / 2=2 p r^{2}$ and $(a-1) / 2=q s^{2}$, then $r$ and $s$ are odd and $b=2 r s \equiv 2(\bmod 4)$. Also, $a=-1+4 p r^{2} \equiv 11(\bmod 32)$. If $(a+1) / 2=p r^{2}$ and $(a-1) / 2=2 q s^{2}$, then again $r$ and $s$ are odd and $b=2 r s \equiv 2$ $(\bmod 4)$. Also, $a=1+4 q s^{2} \equiv-11(\bmod 32)$. Therefore $v_{2}\left(\log _{2} \epsilon\right)=\frac{3}{2}$. It follows that $v_{2}\left(h^{+}\right)=1$.

If $p \equiv q \equiv 3(\bmod 8)$, then $v_{2}\left(h^{-}\right) \geq 3$ by [10, p. 191]. We will show below that $v_{2}\left(h^{+}\right)=1$.

If $v_{2}\left(h^{-}\right) \geq 4$ then $v_{2}\left(b_{0}\right) \geq 3$, so Theorem 1 implies that $3=v_{2}\left(L_{2}(1, \chi)\right)=$ $v_{2}\left(h^{+}\right)+v_{2}\left(\log _{2} \epsilon\right)-\frac{1}{2}=v_{2}\left(\log _{2} \epsilon\right)+\frac{1}{2}$. Therefore $v_{2}\left(\log _{2} \epsilon\right)=\frac{5}{2}$.

If $v_{2}\left(h^{-}\right)=3$ then $4 \leq v_{2}(\beta-1)+3=v_{2}\left(L_{2}(1, \chi)\right)=v_{2}\left(\log _{2} \epsilon\right)+\frac{1}{2}$. Therefore $v_{2}\left(\log _{2} \epsilon\right) \geq \frac{7}{2}$.

In all cases we have $a$ odd and $b$ even. The possibilities $(a+1) / 2=p r^{2},=2 r^{2}$, and $=2 p r^{2}$ are eliminated by congruences mod 8 and the fact that 2 is a quadratic nonresidue $\bmod p$ (and similarly with $q$ in place of $p$ ). Therefore $(a+1) / 2=p q r^{2}$ and $(a-1) / 2=2 s^{2}$. This implies $r$ is odd and $s$ is even. Therefore $a=1+4 s^{2} \equiv 1$ $(\bmod 16)$ and hence $b \equiv 0(\bmod 4)$. This implies that $v_{2}\left(\log _{2} \epsilon\right) \geq \frac{5}{2}$, so we have equality when $v_{2}\left(h^{-}\right) \geq 4$. In general, since $(a+1)(a-1)=2 p q b^{2}$ and $a+1 \equiv 2$ $(\bmod 16)$, we have $\frac{1}{2} v_{2}(a-1)=v_{2}(b)=v_{2}\left(\log _{2} \epsilon\right)-\frac{1}{2}=v_{2}(\beta-1)+2$.

It remains to show that $v_{2}\left(h^{+}\right)=1$. This follows from the work of Rédei and Reichardt [11]; for the convenience of the reader, we reproduce their argument, adapted to the present situation. In the case $v_{2}\left(h^{-}\right) \geq 4$, we note that the desired result follows from $v_{2}\left(\log _{2} \epsilon\right) \geq \frac{5}{2}$ plus the fact that $2 \mid h^{+}$, so we only need to consider the case $v_{2}\left(h^{-}\right)=3$. However, this restriction does not seem to be useful, and we consider the general case. The maximal unramified (including at $\infty$ ) elementary 2extension of $K=\mathbb{Q}(\sqrt{2 p q})$ is $K_{2}=\mathbb{Q}(\sqrt{2}, \sqrt{p q})$, so the 2-class group of $K$ is cyclic. 
Suppose $4 \mid h^{+}$. Then there is a unique unramified extension $K_{4}$ of $K_{2}$ that is cyclic of degree 4 over $K$. Moreover, $\operatorname{Gal}\left(K_{4} / \mathbb{Q}\right)$ is $D_{4}$, the dihedral group of order 8 . Let $I \subseteq \operatorname{Gal}\left(K_{4} / \mathbb{Q}\right)$ be the inertia group for some fixed prime $\mathfrak{p}$ of $K_{4}$ above $p$. Then $I \cap \operatorname{Gal}\left(K_{4} / K\right)=1$. Since $p$ has ramification degree 2 in $\mathbb{Q}(\sqrt{p q}) / \mathbb{Q}$ and in $K_{4} / \mathbb{Q}$, it is unramified in $K_{4} / \mathbb{Q}(\sqrt{p q})$, so $I \cap \operatorname{Gal}\left(K_{4} / \mathbb{Q}(\sqrt{p q})\right)=1$. Therefore $I$ must be one of the two subgroups $\neq \operatorname{Gal}\left(K_{4} / K_{2}\right)$ of order 2 contained in $\operatorname{Gal}\left(K_{4} / \mathbb{Q}(\sqrt{2})\right)$. In particular, $I$ is not normal in $\operatorname{Gal}\left(K_{4} / \mathbb{Q}\right)$. Since $I$ is normal in $Z$, the decomposition group for $\mathfrak{p}, Z$ cannot, be $\operatorname{Gal}\left(K_{4} / \mathbb{Q}\right)$. It follows that $Z$ fixes $\mathbb{Q}(\sqrt{2})$, so $p$ splits in $\mathbb{Q}(\sqrt{2}) / \mathbb{Q}$. Since $p \not \equiv \pm 1(\bmod 8)$, this is a contradiction. Therefore $K_{4}$ does not exist and $v_{2}\left(h^{+}\right)=1$.

In [14], the following was proved. Suppose $q=\left(2^{n}+1\right) / 3$ is prime. Let $m=6 q$. Then $v_{2}(\beta-1) \geq(n-3) / 2$. The last part of the above theorem shows that this is an equality, since $\epsilon=2^{n+1}+1+2^{(n+1) / 2} \sqrt{6 q}$. The conjecture that $\left(2^{n}+1\right) / 3$ is prime infinitely often is discussed in [1].

The remaining cases where $\lambda^{-}=1$ are of the form $\mathbb{Q}(\sqrt{m})$ with $m \equiv 1(\bmod 8)$. Therefore $\chi \omega^{-1}(2)=0$, so the Euler factor disappears in the expression for $L_{2}(0, \chi)$, but $\chi(2)=1$, so the Euler factor $(1-\chi(2) / 2)=1 / 2$ cancels the 2 in the numerator of the formula for $L_{2}(1, \chi)$. Therefore

$$
L_{2}(1, \chi)=\frac{h^{+} \log _{2} \epsilon}{\sqrt{m}} \text { and } v_{2}\left(L_{2}(1, \chi)\right)=v_{2}\left(h^{+}\right)+v_{2}\left(\log _{2} \epsilon\right)
$$

in this case.

Theorem 6. Let $m=p \equiv 9(\bmod 16)$. Then $h^{+}$is odd and $v_{2}\left(h^{-}\right) \geq 2$. Also, $a \equiv 0(\bmod 4), b \equiv 5(\bmod 8)$, and $v_{2}\left(\log _{2} \epsilon\right)=v_{2}(a)$.

1. If $v_{2}\left(h^{-}\right)=2$ then $a \equiv 4(\bmod 8), A \equiv 33(\bmod 256), B \equiv 8(\bmod 16)$, $v_{2}\left(\log _{2} \epsilon\right)=2$, and $L_{2}(s, \chi)$ has no zero in $\mathbb{Z}_{2}$.

2. If $v_{2}\left(h^{-}\right)=3$ then $v_{2}(\beta-1)=v_{2}(a)-3 \geq 1$.

3. If $v_{2}\left(h^{-}\right) \geq 4$ then $v_{2}(a)=3, A \equiv 129(\bmod 1024), B \equiv 16(\bmod 32)$, and $v_{2}(\beta)=v_{2}\left(h^{-}\right)-3 \geq 1$.

Proof. The fact that $h^{+}$is odd is standard. By [5, p. 598], $v_{2}\left(h^{-}\right) \geq 2$. Also, since $p \equiv 1(\bmod 8), a$ and $b$ are integers. Moreover, $a^{2}-p b^{2}=-1$, so $a \equiv 0(\bmod 4)$. Therefore $p b^{2} \equiv 1(\bmod 16)$, and $b \equiv \pm 3(\bmod 8)$. Since -1 is a square $\bmod b$, we must have $b \equiv 1(\bmod 4)$, so $b \equiv 5(\bmod 8)$. Since $\epsilon^{2}=2 a^{2}+1+2 a b \sqrt{p} \equiv 1+2 a b \sqrt{p}$ $(\bmod 4 a), v_{2}\left(\log _{2} \epsilon\right)=v_{2}(a)$.

Assume $v_{2}\left(h^{-}\right)=2$. Then $v_{2}\left(L_{2}(1, \chi)\right)=2$, by Theorem 1 , so $v_{2}(a)=v_{2}\left(\log _{2} \epsilon\right)$ $=2$. The congruences for $A$ and $B$ follow immediately from the fact that $a$ is 4 times an odd number, hence $a^{2} \equiv 16(\bmod 128)$.

Now assume $v_{2}\left(h^{-}\right)=3$. Then there is a zero $\beta$, and $v_{2}(\beta-1)+3=v_{2}(a)=$ $v_{2}\left(\log _{2} \epsilon\right)=v_{2}\left(L_{2}(1, \chi)\right) \geq 4$.

Finally, if $v_{2}\left(h^{-}\right) \geq 4$ then $v_{2}\left(L_{2}(1, \chi)\right)=3$, by the corollary to Theorem 1 . This implies that $v_{2}(a)=v_{2}\left(\log _{2} \epsilon\right)=3$, which yields the desired results.

Theorem 7. Let $m=p q$ with $p \equiv q \equiv \pm 3(\bmod 8)$.

(a) If $p \equiv q \equiv 3(\bmod 8)$ then $v_{2}\left(h^{-}\right)=2, v_{2}\left(h^{+}\right)=0, a \equiv 23(\bmod 64), b \equiv 4$ $(\bmod 8), v_{2}\left(\log _{2} \epsilon\right)=2$, and $L_{2}(s, \chi)$ has no zero in $\mathbb{Z}_{2}$.

(b) If $p \equiv q \equiv 5(\bmod 8)$, then $v_{2}\left(h^{-}\right) \geq 3$.

1. If $v_{2}\left(h^{-}\right)=3$, then $v_{2}\left(h^{+}\right) \geq 1,2 v_{2}(B)-1=v_{2}(A-1) \geq 5$, and $v_{2}(\beta-1)=v_{2}\left(h^{+}\right)+v_{2}(B)-4 \geq 1$. 
2. If $v_{2}\left(h^{-}\right) \geq 4$ then $v_{2}\left(h^{+}\right)=1, v_{2}(\beta)=v_{2}\left(h^{-}\right)-3 \geq 1, v_{2}\left(\log _{2} \epsilon\right)=2$, $A \equiv 33(\bmod 256)$, and $B \equiv 8(\bmod 16)$.

Remark. In part (b)(1), $v_{2}\left(h^{+}\right)$is not constant. For example, if $m=65$ then $h^{-}=8$ and $h^{+}=2$, and if $m=5 \times 461$ then $h^{-}=24$ and $h^{+}=16$.

Proof. (a) $a^{2}-p q b^{2}=1$ implies $a$ is odd and $b$ is even. Therefore we may assume (by switching $p$ and $q$ if necessary) that $(a+1) / 2=p r^{2}$ and $(a-1) / 2=q s^{2}$. This yields $p r^{2}-q s^{2}=1$, hence $r \equiv 2(\bmod 4)$ and $s \equiv 1(\bmod 2)$. Therefore $a=$ $-1+2 p r^{2} \equiv 23(\bmod 64)$. Also, $b=2 r s \equiv 4(\bmod 8)$. Therefore $v_{2}\left(\log _{2} \epsilon\right)=2$. By [10, Prop. 4], $v_{2}\left(h^{-}\right)=2$, so $L_{2}(s, \chi)$ has no zero in $\mathbb{Z}_{2}$ and $v_{2}\left(h^{+}\right)+v_{2}\left(\log _{2} \epsilon\right)=$ $v_{2}\left(L_{2}(1, \chi)\right)=2$. Therefore $v_{2}\left(h^{+}\right)=0$.

(b) By [10, Prop. 4], $v_{2}\left(h^{-}\right) \geq 3$. If $v_{2}\left(h^{-}\right)=3$ then $L_{2}(s, \chi)$ has a zero $\beta \equiv 1$ $(\bmod 2)$. We have $4 \leq v_{2}(\beta-1)+3=v_{2}\left(\log _{2} \epsilon\right)+v_{2}\left(h^{+}\right)$. If $v_{2}\left(h^{-}\right) \geq 4$ then $v_{2}\left(\log _{2} \epsilon\right)+v_{2}\left(h^{+}\right)=v_{2}\left(L_{2}(1, \chi)\right)=3$ and $\beta \equiv 0(\bmod 2)$.

If $\epsilon$ has negative norm, then $a^{2}-p q b^{2}=-1$, so $a \equiv 0(\bmod 4)$, which implies that $A \equiv 1(\bmod 32)$. Since $A^{2}-p q B^{2}=1$, we obtain $B \equiv 0(\bmod 8)$, hence $v_{2}\left(\log _{2} \epsilon\right) \geq 2$. Also, if Norm $\epsilon=-1$ then $h^{+}=h_{0}^{+}$. Since $2 \mid h_{0}^{+}$, we have $v_{2}\left(h^{+}\right) \geq$ 1. In the case that $v_{2}\left(h^{-}\right) \geq 4$, we therefore obtain $v_{2}\left(\log _{2} \epsilon\right)=2$ and $v_{2}\left(h^{+}\right)=1$.

If $\epsilon$ has positive norm, then $a^{2}-p q b^{2}=+1$, so $a$ is odd and $b \equiv 0(\bmod 4)$. We may assume $(a+1) / 2=p r^{2}$ and $(a-1) / 2=q s^{2}$, which yields $p r^{2}-q s^{2}=1$. Therefore $p$ is a square $\bmod q$ (and $q$ is a square $\bmod p$ ), so $4 \mid h_{0}^{+}$(see [5, p. 596]). Therefore $v_{2}\left(h^{+}\right) \geq 1$. Again in the case that $v_{2}\left(h^{-}\right) \geq 4$, we obtain $v_{2}\left(\log _{2} \epsilon\right)=2$ and $v_{2}\left(h^{+}\right)=1$.

When $v_{2}\left(\log _{2} \epsilon\right)=2$, in both cases $(\mathrm{N} \epsilon=+1$ and $\mathrm{N} \epsilon=-1)$ we have $B \equiv 8$ $(\bmod 16)$, so $A^{2} \equiv 65(\bmod 512)$. Therefore $A \equiv \pm 33(\bmod 256)$. Since $A=2 a^{2}-$ $\mathrm{N} \epsilon=2 p q b^{2}+\mathrm{N} \epsilon \equiv 1(\bmod 32)$ in both cases, we obtain $A \equiv 33(\bmod 256)$.

Finally, for completeness, we list what happens when $\lambda^{-}=0$. The proofs, which we omit, are very similar to those given above. Note that in this case we have both $v_{2}\left(b_{0}\right)=0$ and $v_{2}\left(L_{2}(1, \chi)\right)=1$, so we expect congruences for $h^{-}, h^{+}$, and $\epsilon$.

In part (2), we consider $\epsilon^{3}$ instead of $\epsilon$, since $\epsilon$ is not necessarily in $\mathbb{Z}[\sqrt{p}]$. Note that $v_{2}\left(\log _{2}\left(\epsilon^{3}\right)\right)=v_{2}\left(\log _{2} \epsilon\right)$, so there is little effect on our other calculations.

Theorem 8. Suppose $\lambda^{-}=0$.

1. If $m=p \equiv 3(\bmod 8)$, then $v_{2}\left(h^{-}\right)=0, v_{2}\left(h^{+}\right)=0, v_{2}\left(\log _{2} \epsilon\right)=1, a \equiv 2$ $(\bmod 4), A \equiv 7(\bmod 64), B \equiv 4(\bmod 8)$.

2. If $m=p \equiv 5(\bmod 8)$, then $v_{2}\left(h^{-}\right)=1, v_{2}\left(h^{+}\right)=0, v_{2}\left(\log _{2} \epsilon\right)=1$. If $\epsilon^{3}=a^{\prime}+b^{\prime} \sqrt{p}$ and $\epsilon^{6}=A^{\prime}+B^{\prime} \sqrt{p}$, then $a^{\prime} \equiv 2(\bmod 4), b^{\prime} \equiv 1(\bmod 4)$, $A^{\prime} \equiv 9(\bmod 64), B^{\prime} \equiv 4(\bmod 8)$.

3. If $m=2 p$ with $p \equiv 3(\bmod 8)$, then $v_{2}\left(h^{-}\right)=1, v_{2}\left(h^{+}\right)=0, v_{2}\left(\log _{2} \epsilon\right)=\frac{3}{2}$, $a \equiv 5(\bmod 32), b \equiv 2(\bmod 4)$.

4. If $m=2 p$ with $p \equiv 5(\bmod 8)$, then $v_{2}\left(h^{-}\right)=1, v_{2}\left(h^{+}\right)=1, v_{2}\left(\log _{2} \epsilon\right)=\frac{1}{2}$, $a \equiv 1(\bmod 2), b \equiv 1(\bmod 2)$.

\section{Numerical Results}

Using PARI, we calculated $v_{2}\left(h^{+} \log _{2} \epsilon\right)$ and $v_{2}\left(h^{-}\right)$, and consequently $v_{2}(\beta-1)$ and $v_{2}(\beta)$, and obtained the following data. For example, the 311 in the first row of the first table means that there are 311 primes $p \equiv 9(\bmod 16)$ less than $10^{5}$ such that there is a zero $\beta$ with $v_{2}(\beta-1)=0$ for the 2 -adic $L$-function of the 


\begin{tabular}{|c|c|c|c|c|c|c|c|c|c|}
\hline \multicolumn{10}{|c|}{$\mathbb{Q}(\sqrt{ } 2 p)$ with $p \equiv 9(\bmod 10$} \\
\hline$v_{2}(\beta-1)$ & no $\beta$ & 0 & 1 & 2 & 3 & 4 & 5 & 6 & $\geq 7$ \\
\hline $0<p<10^{5}$ & 601 & 311 & 160 & 74 & 26 & 12 & 7 & 3 & 2 \\
\hline $10^{5}<p<2 \times 10^{5}$ & 519 & 252 & 128 & 82 & 28 & 15 & 10 & 3 & 4 \\
\hline $2 \times 10^{5}<p<3 \times 10^{5}$ & 489 & 251 & 139 & 63 & 28 & 12 & 7 & 3 & 1 \\
\hline $3 \times 10^{5}<p<4 \times 10^{5}$ & 498 & 243 & 106 & 56 & 28 & 11 & 10 & 6 & 3 \\
\hline $4 \times 10^{5}<p<5 \times 10^{5}$ & 477 & 236 & 123 & 74 & 37 & 10 & 10 & 3 & 4 \\
\hline Total & 2584 & 1293 & 656 & 349 & 147 & 60 & 44 & 18 & 14 \\
\hline
\end{tabular}

\begin{tabular}{|r|r|c|c|c|c|r|r|r|}
\hline$v_{2}(\beta)$ & \multicolumn{1}{|c|}{0} & 1 & \multicolumn{1}{c|}{2} & \multicolumn{1}{c|}{3} & 4 & \multicolumn{1}{c|}{5} & \multicolumn{1}{|c|}{6} & $\geq 7$ \\
\hline $0<p<10^{5}$ & 284 & 155 & 79 & 41 & 24 & 12 & 0 & 0 \\
$10^{5}<p<2 \times 10^{5}$ & 270 & 134 & 50 & 30 & 13 & 20 & 5 & 0 \\
$2 \times 10^{5}<p<3 \times 10^{5}$ & 253 & 132 & 58 & 31 & 13 & 12 & 5 & 0 \\
$3 \times 10^{5}<p<4 \times 10^{5}$ & 220 & 137 & 51 & 32 & 10 & 6 & 3 & 4 \\
$4 \times 10^{5}<p<5 \times 10^{5}$ & 261 & 120 & 59 & 30 & 12 & 6 & 5 & 4 \\
Total & 1288 & 678 & 297 & 164 & 72 & 56 & 18 & 8 \\
\hline
\end{tabular}

$\mathbb{Q}(\sqrt{6 q})$ with $q \equiv 3(\bmod 8) \quad($ cf. Theorem 5 , part $(3))$

\begin{tabular}{|r|r|r|r|r|r|r|r|r|}
\hline$v_{2}(\beta-1)$ & \multicolumn{1}{|c|}{0} & \multicolumn{1}{|c|}{1} & \multicolumn{1}{c|}{2} & \multicolumn{1}{c|}{3} & \multicolumn{1}{|c|}{5} & \multicolumn{1}{c|}{6} & $\geq 7$ \\
\hline $3<q<10^{5}$ & 1192 & 582 & 329 & 144 & 76 & 39 & 21 & 25 \\
$10^{5}<q<2 \times 10^{5}$ & 1052 & 493 & 271 & 128 & 67 & 30 & 15 & 30 \\
$2 \times 10^{5}<q<3 \times 10^{5}$ & 1005 & 493 & 223 & 147 & 62 & 41 & 23 & 5 \\
$3 \times 10^{5}<q<4 \times 10^{5}$ & 978 & 523 & 250 & 95 & 70 & 25 & 22 & 12 \\
$4 \times 10^{5}<q<5 \times 10^{5}$ & 976 & 456 & 249 & 143 & 57 & 41 & 14 & 13 \\
Total & 5203 & 2547 & 1322 & 657 & 332 & 176 & 95 & 85 \\
\hline
\end{tabular}

\begin{tabular}{|r|r|r|r|c|c|r|r|r|}
\hline$v_{2}(\beta)$ & \multicolumn{1}{|c|}{0} & \multicolumn{1}{c|}{1} & \multicolumn{1}{c|}{2} & 3 & \multicolumn{1}{c|}{4} & \multicolumn{1}{c|}{5} & \multicolumn{1}{c|}{6} & $\geq 7$ \\
\hline $3<q<10^{5}$ & 1216 & 575 & 304 & 158 & 87 & 47 & 21 & 0 \\
$10^{5}<q<2 \times 10^{5}$ & 1034 & 521 & 260 & 144 & 61 & 24 & 35 & 7 \\
$2 \times 10^{5}<q<3 \times 10^{5}$ & 994 & 497 & 262 & 118 & 64 & 37 & 17 & 10 \\
$3 \times 10^{5}<q<4 \times 10^{5}$ & 997 & 492 & 258 & 119 & 63 & 30 & 9 & 7 \\
$4 \times 10^{5}<q<5 \times 10^{5}$ & 973 & 474 & 243 & 126 & 73 & 27 & 15 & 18 \\
Total & 5214 & 2559 & 1327 & 665 & 348 & 165 & 97 & 42 \\
\hline
\end{tabular}

corresponding quadratic field $\mathbb{Q}(\sqrt{2 p})$. Note that this number also gives the total number of examples of $v_{2}(\beta)>0$ (i.e., the sum of the first row of the second part of the table, omitting the first entry).

These tables indicate that, for the fields considered such that $\beta$ exists, $v_{2}(\beta-1)=$ $i \geq 0$ with probability approximately $2^{-(i+1)}$. Similarly, $v_{2}(\beta)=i \geq 0$ with probability approximately $2^{-(i+1)}$. In the families where $\beta$ does not always exist, approximately half of the fields are such that $\beta$ exists.

The 2-parts of the class groups of the imaginary quadratic fields considered are either cyclic $\left(2^{j}\right)$ with $j \geq 2$ (for $\mathbb{Q}(\sqrt{-2 p})$ and $\mathbb{Q}(\sqrt{-p})$ ) or of the form $(2) \times\left(2^{j}\right)$ with $j \geq 2$ (for $\mathbb{Q}(\sqrt{-6 q})$ and $\mathbb{Q}(\sqrt{-5 p})$. This follows from [11]). The philosophy of the Cohen-Lenstra heuristics [3], extended to the present situation, would predict that the occurrence of a group as the 2-part of the class group is inversely proportional to the size of its automorphism group. For the case where the 2-part 


\begin{tabular}{|c|c|c|c|c|c|c|c|c|c|}
\hline \multicolumn{10}{|c|}{$\mathbb{Q}(\sqrt{p})$ with $p \equiv 9(\bmod 16)$} \\
\hline$v_{2}(\beta-1)$ & no $\beta$ & 0 & 1 & 2 & 3 & 4 & 5 & 6 & $\geq 7$ \\
\hline $0<p<10^{5}$ & 595 & 309 & 127 & 88 & 43 & 15 & 9 & 7 & 3 \\
\hline $10^{5}<p<2 \times 10^{5}$ & 522 & 258 & 128 & 69 & 35 & 14 & 9 & 3 & 3 \\
\hline $2 \times 10^{5}<p<3 \times 10^{5}$ & 504 & 241 & 128 & 52 & 34 & 19 & 11 & 3 & 1 \\
\hline $3 \times 10^{5}<p<4 \times 10^{5}$ & 463 & 256 & 122 & 63 & 27 & 19 & 6 & 3 & 2 \\
\hline $4 \times 10^{5}<p<5 \times 10^{5}$ & 497 & 239 & 119 & 64 & 34 & 12 & 5 & 2 & 2 \\
\hline Total & 2581 & 1303 & 624 & 336 & 173 & 79 & 40 & 18 & 11 \\
\hline
\end{tabular}

\begin{tabular}{|r|r|c|r|r|r|r|r|}
\hline$v_{2}(\beta)$ & \multicolumn{1}{|c|}{0} & 1 & \multicolumn{1}{|c|}{2} & \multicolumn{1}{|c|}{3} & \multicolumn{1}{|c|}{4} & \multicolumn{1}{c|}{5} & \multicolumn{1}{c|}{6} \\
\hline $0<p<10^{5}$ & 292 & 153 & 93 & 35 & 22 & 5 & 1 \\
$10^{5}<p<2 \times 10^{5}$ & 261 & 139 & 64 & 25 & 15 & 8 & 7 \\
$2 \times 10^{5}<p<3 \times 10^{5}$ & 248 & 132 & 55 & 28 & 9 & 9 & 8 \\
$3 \times 10^{5}<p<4 \times 10^{5}$ & 242 & 128 & 72 & 29 & 17 & 7 & 3 \\
$4 \times 10^{5}<p<5 \times 10^{5}$ & 238 & 123 & 58 & 29 & 13 & 6 & 10 \\
Total & 1281 & 675 & 342 & 146 & 76 & 35 & 29 \\
\hline
\end{tabular}

\begin{tabular}{|c|c|c|c|c|c|c|c|c|}
\hline$v_{2}(\beta-1)$ & 0 & 1 & 2 & 3 & 4 & 5 & 6 & $>7$ \\
\hline $5<p<10^{5}$ & 1192 & 620 & 306 & 148 & 58 & 40 & 14 & 20 \\
\hline $10^{5}<p<2 \times 10^{5}$ & 1061 & 513 & 275 & 136 & 68 & 29 & 17 & 13 \\
\hline $2 \times 10^{5}<p<3 \times 10^{5}$ & 1001 & 510 & 270 & 91 & 69 & 36 & 17 & 18 \\
\hline $3 \times 10^{5}<p<4 \times 10^{5}$ & 1004 & 492 & 235 & 137 & 49 & 25 & 15 & 16 \\
\hline $4 \times 10^{5}<p<5 \times 10^{5}$ & 940 & 475 & 254 & 122 & 53 & 30 & 13 & 14 \\
\hline Total & 5198 & 2610 & 1340 & 634 & 297 & 160 & 76 & 81 \\
\hline
\end{tabular}

\begin{tabular}{|r|r|r|r|c|c|r|r|r|}
\hline$v_{2}(\beta)$ & \multicolumn{1}{|c|}{0} & \multicolumn{1}{|c|}{1} & \multicolumn{1}{c|}{2} & 3 & \multicolumn{1}{|c|}{4} & \multicolumn{1}{c|}{5} & 6 & $\geq 7$ \\
\hline $5<p<10^{5}$ & 1206 & 601 & 300 & 152 & 76 & 44 & 18 & 1 \\
$10^{5}<p<2 \times 10^{5}$ & 1051 & 526 & 276 & 120 & 85 & 27 & 17 & 10 \\
$2 \times 10^{5}<p<3 \times 10^{5}$ & 1011 & 487 & 248 & 136 & 64 & 29 & 23 & 14 \\
$3 \times 10^{5}<p<4 \times 10^{5}$ & 969 & 458 & 253 & 153 & 81 & 32 & 17 & 10 \\
$4 \times 10^{5}<p<5 \times 10^{5}$ & 961 & 463 & 228 & 124 & 66 & 35 & 16 & 8 \\
Total & 4898 & 2535 & 1305 & 685 & 372 & 167 & 91 & 43 \\
\hline
\end{tabular}

of the class group is cyclic of order $2^{j}$, the automorphism group has order $2^{j-1}$. Combining this with the above, we find that this extension of the Cohen-Lenstra heuristics to these cases is equivalent to the statement that $v_{2}(\beta)=i \geq 0$ with probability $2^{-(i+1)}$.

The elements of the automorphism group of $(2) \times\left(2^{j}\right)$ with $j \geq 2$ can be represented by matrices $\left(\begin{array}{ll}a & b \\ c & d\end{array}\right)$, where $a=1, b \in \operatorname{Hom}\left(\mathbb{Z} / 2 \mathbb{Z}, \mathbb{Z} / 2^{j} \mathbb{Z}\right), c \in$ $\operatorname{Hom}\left(\mathbb{Z} / 2^{j} \mathbb{Z}, \mathbb{Z} / 2 \mathbb{Z}\right)$, and $d \in \operatorname{Aut}\left(\mathbb{Z} / 2^{j} \mathbb{Z}\right)$. Therefore this automorphism group has order $2^{j+1}$. Again we find that the extension of the Cohen-Lenstra heuristics is equivalent to $v_{2}(\beta)=i$ with probability $2^{-(i+1)}$. 


\section{REFERENCES}

1. P. T. Bateman, J. L. Selfridge, and S. S. Wagstaff, Jr., The new Mersenne conjecture, Am. Math. Monthly 96 (1989), 125-128. MR 90c:11009

2. N. Childress and R. Gold, Zeros of p-adic L-functions, Acta Arith. 48 (1987), 63-71. MR 88i: 11091

3. H. Cohen and H. W. Lenstra, Jr., Heuristics on class groups of number fields, Number Theory, Noordwijkerhout 1983, 33-62, Springer Lecture Notes in Math. 1068 (1984). MR 85j:11144

4. B. Ferrero, The cyclotomic $\mathbb{Z}_{2}$-extension of imaginary quadratic fields, Amer. J. Math. 102 (1980), 447-459. MR 81g:12006

5. P. Kaplan, Divisibilité par 8 du nombre des classes des corps quadratiques dont le 2-groupe des classes est cyclique, et réciprocité biquadratique, J. Math. Soc. Japan 25 (1973), 596-608. MR 48:2113

6. Y. Kida, On cyclotomic $\mathbb{Z}_{2}$-extensions of imaginary quadratic fields, Tôhoku Math. J. (2), 31 (1979), 91-96. MR 80d:12003

7. F. Morain, e-mail announcement, April 29, 1996.

8. P. Morton, The quadratic number fields with cyclic 2-classgroups, Pacific J. Math. 108 (1983), 165-175. MR 84i:12001

9. C. D. Olds, Continued Fractions, Random House, New York, 1963. MR 26:3672

10. A. Pizer, On the 2-part of the class number of imaginary quadratic number fields, J. Number Theory 8 (1976), 184-192. MR 53:10759

11. L. Rédei and H. Reichardt, Die Anzahl der durch 4 teilbaren Invarianten der Klassengruppe eines beliebigen quadratisches Zahlkörpers, J. reine angew. Math. 170 (1934), 69-74.

12. L. Washington, Zeros of p-adic L-functions, Sém. Théorie des Nombres, Paris 1980-1981, Birkhäuser (1982), 337-357. MR 84f:12008

13. L. Washington, Introduction to cyclotomic fields, Springer-Verlag, New York-Berlin, 1982. MR 85g:11001

14. L. Washington, Siegel zeros for 2-adic L-functions, Number theory (Halifax, NS, 1994) CMS Conf. Proc., vol. 15, Amer. Math. Soc. (1995), 393-396. MR 96k:11145

15. L. Washington, A family of cubic fields and zeros of 3-adic L-functions, J. Number Theory 63(1997), 408-417. MR 98e:11126

Department of Mathematics, University of Maryland, College Park, MD 20742

Department of Mathematics \& Comp. Sci., Caldwell College, Caldwell, NJ 07006

E-mail address: PSime@caldwell.edu

Department of Mathematics, University of Maryland, College Park, MD 20742

E-mail address: lcw@math.umd.edu 